\title{
Frequency and Types of Hyponatremia in Stroke Patients Admitted in a Referral Neuroscience Institute of Dhaka
}

\author{
Maliha Hakim*, Mashfiqul-Hasan, Mahmudul Islam, Mohammad Akter Hossain, Jobaida Naznin, \\ Saifur Rahman Khan
}

Department of Neurology, National Institute of Neurosciences and Hospital, Dhaka, Bangladesh

Email address:

drmalihahakim@yahoo.com (M. Hakim)

${ }^{*}$ Corresponding author

\section{To cite this article:}

Maliha Hakim, Mashfiqul-Hasan, Mahmudul Islam, Mohammad Akter Hossain, Jobaida Naznin, Saifur Rahman Khan. Frequency and Types of Hyponatremia in Stroke Patients Admitted in a Referral Neuroscience Institute of Dhaka. Clinical Neurology and Neuroscience.

Vol. 3, No. 2, 2019, pp. 46-49. doi: 10.11648/j.cnn.20190302.14

Received: May 8, 2019; Accepted: June 11, 2019; Published: June 20, 2019

\begin{abstract}
Background: Hyponatremia is a common electrolyte abnormality in acute stroke patients and may be related to variable etiology. Objective: To observe the frequency and types of hyponatremia in hospitalized acute stroke patients. Materials and methods: This cross-sectional study, carried out in a referral neuroscience institute of Dhaka during February to November 2017, included 209 patients admitted with acute stroke (65 ischemic, age $61.5 \pm 13.3$ years, M/F: 45/20; 144 hemorrhagic, age 59.2 \pm 13.1 years, M/F: 80/64). The clinical and laboratory values on admission were recorded. Those having hyponatremia (serum sodium $<135 \mathrm{mmol} / \mathrm{L}$ ) on admission were evaluated by clinical features (history of vomiting or diarrhoea, volume status, urine output) and laboratory parameters (urine osmolality, urine sodium, plasma osmolality, blood urea, hematocrit) to determine the types of hyponatremia. Results: Four patients died before the serum could be sent for electrolytes. Among the rest, 36 (17.6\%) had hyponatremia on admission. Serum sodium level was $<125 \mathrm{mmol} / \mathrm{L}$ in $7(19.4 \%)$ and $125-134 \mathrm{mmol} / \mathrm{L}$ in rest of the patients having hyponatremia $(29 ; 80.6 \%)$. The frequency of hyponatremia was similar in ischemic and hemorrhagic stroke $(17.2 \%$ vs. $17.7 \%, \mathrm{p}=0.925)$. Syndrome of inappropriate antidiuresis (SIAD) was most frequent cause of hyponatremia (50.0\%), followed by cerebral salt wasting (CSW; 30.6\%). The rest had either hyponatremia related to gastrointestinal (GI) fluid loss $(2.8 \%)$ or died before a cause of hyponatremia could be ascertained (11.1\%). There was no significant difference of age, gender, NIHSS score and GCS score on admission as well as in hospital stay and inhospital mortality between patients with or without hyponatremia ( $\mathrm{p}=\mathrm{ns}$ for all). Frequency of CSW was relatively higher in hemorrhagic stroke (hemorrhagic vs. ischemic: $32.0 \%$ vs. $27.3 \%$ ) and SIAD in ischemic stroke (hemorrhagic vs. ischemic: $40.0 \%$ vs. $72.7 \%$ ) but did not reach level of statistical significance. Conclusion: Frequency of hyponatremia seems remarkable in hospitalized acute stroke patients, SIAD and CSW being the most frequent cause.
\end{abstract}

Keywords: Hyponatremia, Stroke, Dhaka

\section{Introduction}

Hyponatremia is a common finding in critically ill patients; acute stroke is no exception [1]. Usually, neurological insult itself leads to either syndrome of inappropriate anti-diuresis (SIAD) or cerebral salt wasting (CSW) in patients with stroke, but other common causes like gastrointestinal fluid loss or drug related causes may also be present in such condition. Differentiation of these conditions is important as the modalities and mood of correcting hyponatremia is different [2]. Along with clinical picture, plasma and urinary osmolality and sodium often guides to the cause of hyponatremia [3].

There is no absolute laboratory parameter that is able to differentiate SIAD and CSW. Rather, a group of clinical features like volume status, hemodynamics and fluid balance are helpful in this regard. Some supportive lab features that include blood urea, uric acid, hematocrit etc. are used to strengthen the clinical suspicion [4]. The timely and proper correction of hyponatremia in acutely ill stroke patients may improve the outcome and reduce the hospital stay. So, to 
assess the frequency as well as the types of hyponatremia in acute stroke patients the current study was carried out in a referral neuroscience hospital of Dhaka, Bangladesh.

\section{Materials and Methods}

\subsection{Study Subjects and Design}

This cross-sectional study, carried out from February to November 2017 in Neurology department of National Institute of Neurosciences (NINS) and Hospital, Dhaka, Bangladesh, included 209 patients admitted with acute stroke (65 ischemic; age 61.5 13.3 years, M/F: 45/20; 144 hemorrhagic, age $59.2 \pm 13.1$ years, M/F: 80/64) by convenient sampling. The clinical and laboratory values on admission as well as length of hospital stay and in-hospital mortality were recorded.

Those having hyponatremia (serum sodium $<135 \mathrm{mmol} / \mathrm{L}$ ) on admission were evaluated by clinical evaluation (history of vomiting or diarrhoea, volume status, urine output) and laboratory parameters (urine osmolality, urine sodium, plasma osmolality, blood urea, hematocrit) to determine the type of hyponatremia according to the current recommendations (Figure 1) [4, 5].

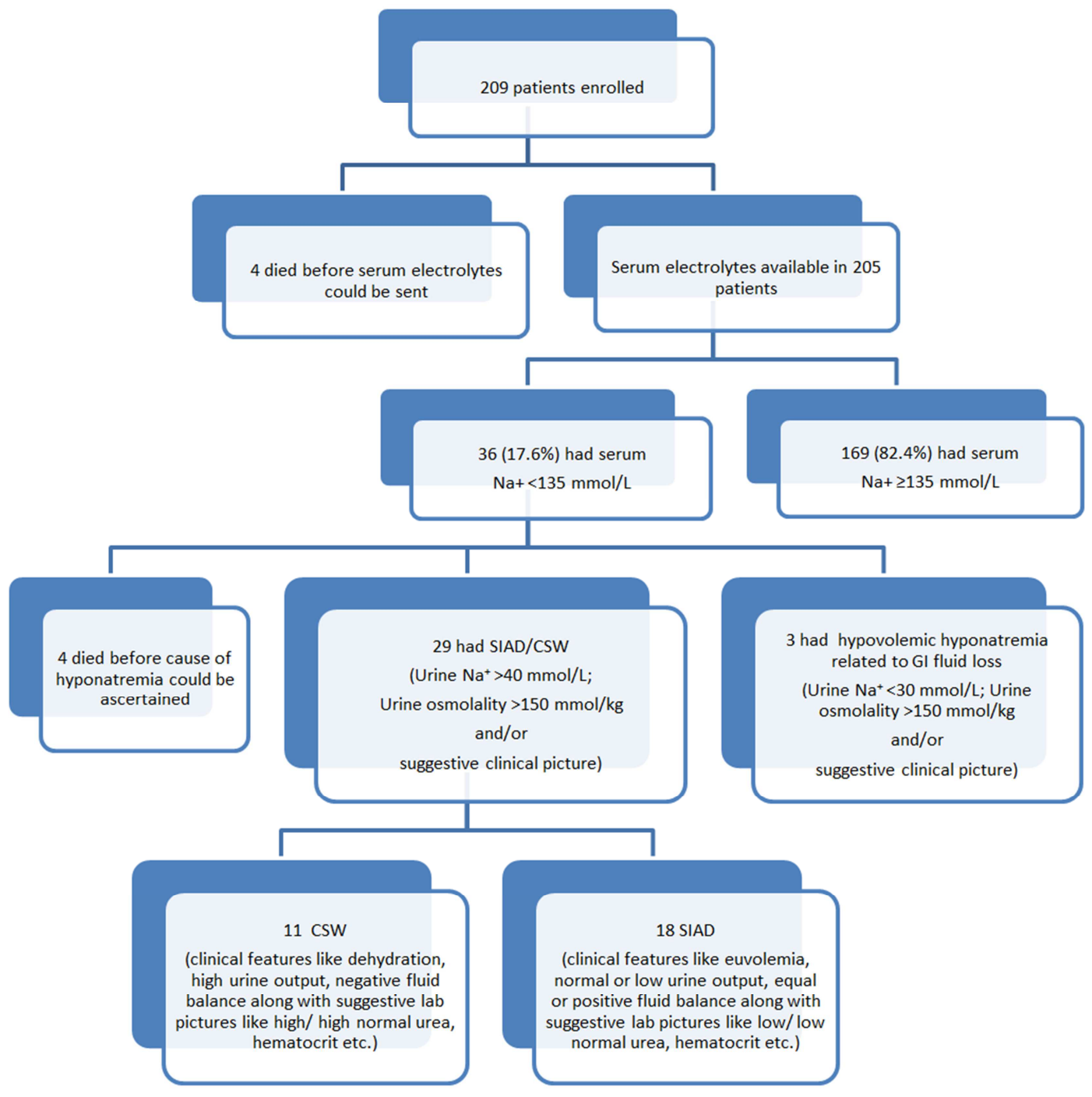

Figure 1. Flow chart showing the patient recruitment and study procedure.

\subsection{Statistical Analysis}

Categorical data were expressed as frequencies and percentages, while numeric data were expressed as mean $( \pm$ SD) when normally distributed and as median and interquartile range (IQR; $25^{\text {th }}-75^{\text {th }}$ percentile) when skewed. Assessment of normality of data was done by Shapiro-Wilk test. Comparison of categorical variable was done by $\chi 2$-test and numeric variable by unpaired t-test or Mann-Whitney U test as applicable. $\mathrm{P}$ values $<0.05$ were considered as significant. SPSS (version 22.0) was used for data analysis.

\subsection{Ethical Aspects}

Written informed consent was taken from the attendants of the participants. The project was run after approval of the institutional review board (IRB) of NINS. 


\section{Results}

Four patients died before the serum could be sent for electrolytes. Among the rest, $36(17.6 \%)$ had hyponatremia on admission. Serum sodium level was $<125 \mathrm{mmol} / \mathrm{L}$ in 7 $(19.4 \%)$ and $125-134 \mathrm{mmol} / \mathrm{L}$ in rest of the patients having hyponatremia $(29,80.6 \%)$. The frequency of hyponatremia was similar in ischemic and hemorrhagic stroke (Table 1). Comparison of clinical characteristics of patients with or without hyponatremia revealed that, there was no significant difference between these groups in relation to age, gender, National Institute of Health Stroke Scale (NIHSS) score, Glasgow Coma Scale (GCS) score, length of hospital stay and in-hospital mortality (Table 2). Median serum sodium was 130 (IQR 125-133) in hyponatremia group and 140 (IQR 138-143) in normal serum sodium group.

Table 1. Frequency of hyponatremia in stroke patients.

\begin{tabular}{llll}
\hline \multirow{2}{*}{ Type of stroke } & \multicolumn{2}{l}{ Hyponatremia } & \multirow{2}{*}{$* \mathbf{p}$} \\
\cline { 2 - 3 } & Absent & Present & \multirow{2}{*}{0.925} \\
\hline Ischemic $(\mathrm{n}=64)$ & $53(82.8 \%)$ & $11(17.2 \%)$ & \\
Hemorrhagic $(\mathrm{n}=131)$ & $116(82.3 \%)$ & $25(17.7 \%)$ & \\
Total & $169(82.4 \%)$ & $36(17.6 \%)$ & \\
\hline
\end{tabular}

*by $\chi 2$ test

Within parentheses are percentages over row total

Table 2. Comparison of clinical characteristics of patients with or without hyponatremia.

\begin{tabular}{|c|c|c|c|c|}
\hline Characters & Hyponatremia group $(n=36)$ & Normal sodium group $(n=169)$ & All patients $(\mathrm{N}=\mathbf{2 0 5})$ & p-value \\
\hline Age (years; mean \pm SD) & $63.4 \pm 10.5$ & $59.2 \pm 13.5$ & $60.0 \pm 13.1$ & 0.09 \\
\hline Female sex & $13(36.1)$ & $68(40.2)$ & $81(39.5)$ & 0.65 \\
\hline NIHSS score (median \& IQR) & $21(11-26)$ & $18(10-26)$ & $19(11-26)$ & 0.54 \\
\hline GCS score (median \& IQR) & $10(7-12)$ & $9(7-13)$ & $10(7-13)$ & 0.66 \\
\hline Sodium (mmol/L; median \& IQR) & $130(125-133)$ & $140(138-143)$ & $139(136-142)$ & $<0.001$ \\
\hline Length of hospital stay (days; mean \pm SD) & $6.9 \pm 3.2$ & $6.0 \pm 3.2$ & $6.2 \pm 3.2$ & 0.11 \\
\hline In-hospital mortality & $6(16.7)$ & $29(17.2)$ & $35(17.1)$ & 0.94 \\
\hline
\end{tabular}

P-value stands for difference between hyponatremia group and normal soidum group

Within parentheses are percentages over column total, if not mentioned otherwise

SIAD was most frequent cause of hyponatremia (50.0\%), followed by CSW (30.6\%). The rest had either hyponatremia related to gastrointestinal (GI) fluid loss $(8.3 \%)$ or died before a cause of hyponatremia could be ascertained (11.1\%; Figure 2). Frequency of CSW was relatively higher in hemorrhagic stroke (hemorrhagic vs. ischemic: $32.0 \%$ vs. $27.3 \%$ ) and SIAD in ischemic stroke (hemorrhagic vs. ischemic: $40.0 \%$ vs. $72.7 \%$ ) but did not reach level of statistical significance (Table 3).

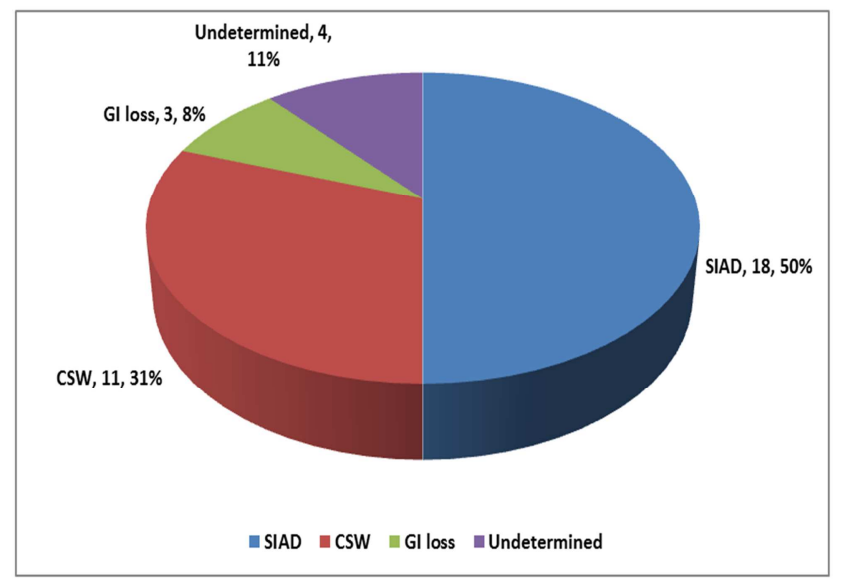

Figure 2. Frequency of different causes of hyponatremia in acute stroke $(n=36)$.

SIAD: Syndrome of inappropriate antidiuresis

CSW: Cerebral salt wasting

GI: Gastrointestinal
Table 3. Types of hyponatremia in different strokes.

\begin{tabular}{llll}
\hline $\begin{array}{l}\text { Types of } \\
\text { hyponatremia }\end{array}$ & Ischemic stroke & Hemorrhagic stroke & *p \\
\hline CSW & $3(27.3)$ & $8(32.0)$ & \\
SIAD & $8(72.7)$ & $10(40.0)$ & 0.19 \\
GI loss & $0(0.0)$ & $3(12.0)$ & \\
Undetermined & $0(0.0)$ & $4(16.0)$ & \\
Total & 11 & 25 & \\
\hline
\end{tabular}

*by $\chi 2$ test

Within parentheses are percentages over column total

SIAD: Syndrome of inappropriate antidiuresis

CSW: Cerebral salt wasting

GI: Gastrointestinal

\section{Discussions}

In this study, we observed that nearly 1 in 5 patients admitted with acute stroke in a tertiary level hospital of Dhaka city had hyponatremia; frequency of which was similar in both hemorrhagic and ischemic stroke patients. SIAD was observed to be the most frequent underlying cause. Frequency of CSW was also remarkable.

In various studies, frequency of hyponatremia was observed to be $10-40 \%$ [6-12]. The variability in the frequency may reflect different case definitions (eg. serum sodium level $<135$ or $<130$ ) and clinical diversity of the patients in different studies. Our study was conducted in the in-patient department of a referral neurology institute, where severe cases are referred from all over the country. So, although our findings reflect the whole country, less severe stroke cases are not included in this study. The median NIHSS score of the patients also suggests the same.

It is important to look for the presence of hyponatremia because it not only affects the short term clinical course, but is also regarded as an independent predictor of mortality in 
both hemorrhagic and ischemic stroke $[10,15]$. We did not observe any significant difference in in-hospital mortality in stroke patients with hyponatremia; neither has it prolonged the hospital stay. One explanation may be related to severity of hyponatremia, as most of our participants having hyponatremia had serum sodium between 125 and 134 $\mathrm{mmol} / \mathrm{L}$. It is possible that more intense hyponatremia may affect the outcome more pronouncedly. Prospective studies with larger sample size may be required to observe the effect of hyponatremia on mortality.

The mechanism of hyponatremia in stroke patient may be diverse. But, apart from hypovolemic hyponatremia related to gastrointestinal fluid loss, SIAD and CSW is regarded as most important cause of hyponatremia in stroke. Despite having some overlapping features, two conditions can be separated by careful clinical consideration with supportive laboratory findings. Pinpointing the cause of hyponatremia can modify the treatment approach [7]. Although our study did not observe any statistical significant difference in frequency of SIAD and CSW in different types of stroke, SIAD was more frequent in ischemic stroke and CSW in hemorrhagic stroke. Further studies are required to validate these findings.

\section{Conclusion}

Frequency of hyponatremia seems remarkable in patients hospitalized for both hemorrhagic and ischemic acute stroke. SIAD and CSW are observed to be the most frequent cause.

\section{Acknowledgements}

The authors are grateful to the supporting staffs and colleagues of the department.

\section{Conflict of Interest}

There is no conflict of interest relevant to this paper to disclose.

\section{Funding Agency}

This research project was funded by Bangladesh Medical Research Council (BMRC).

\section{References}

[1] Padhi R, Panda BN, Jagati S, Patra SC. Hyponatremia in critically ill patients. Indian J Crit Care Med. 2014; 18 (2): 8387.
[2] Sterns RH, Silver SM. Cerebral salt wasting versus SIADH: What difference? (Clinical commentary). J Am Soc Nephrol 2008; 19: 194-96.

[3] Zomp A, Alexander E. Syndrome of inappropriate antidiuretic hormone and cerebral salt wasting in critically ill patients. AACN Adv Crit Care. 2012; 23 (3): 233-39

[4] Albanese A, Hindmarsh P, Stanhope R. Management of hyponatremia in patients with acute cerebral insults. Arch Dis Child 2001; 85: 246-51.

[5] Natarajan K, Prasad M. Hyponatremia in stroke: cerebral salt wasting versus syndrome of inappropriate anti-diuresis. IOSRJDMS 2016; 15: 1-11.

[6] Saleem S, Yousuf I, Gul A, Gupta S, Verma S. Hyponatremia in stroke. Ann Indian Acad Neurol. 2014; 17 (1): 55-57.

[7] Alam MN, Uddin MJ, Rahman KM, Ahmed S, Akhter M et al. Electrolyte changes in stroke. Mymensingh Med J. 2012; 21 (4): 594-99.

[8] Bandyopadhyay M, Jatua SK, Adhikari M, Bhandari A. Study of electrolyte abnormality in acute stroke. Ann. Int. Med. Den. Res. 2017; 3 (5): 4-9.

[9] Siddiqui MR, Islam QT, Haque MA, Iqbal MJ, Hossain A et al. Dyselectrolytaemia in acute stroke patients, an observational study. Bangladesh J Medicine. 2011; 22: 30-34.

[10] Kuramatsu JB, Bobinger T, Volbers B, Staykov D, Lucking H et al. Hyponatremia is an independent predictor of in-hospital mortality in spontaneous intracerebral hemorrhage. Stroke. 2014; 45: 1285-91.

[11] Kembuan MAHN, Sekeon SAS. Electrolyte disturbances among acute stroke patients in Manado, Indonesia. GJMEDPH. 2014; 3 (1).

[12] Amin S. Haq A, Faraz M, Wazir MN. Syndrome of inappropriate antidiuretic hormone secretion and neurological stroke. KJMS. 2014; 7 (1): 162-65.

[13] Karunanandham S, Rajappa T, Selvaraju K. Hyponatremia in Patients Admitted with Stroke. Journal of Clinical \& Diagnostic Research. 2018 Aug 1; 12 (8).

[14] Rodrigues B, Staf I, Fortunato G, McDullouhg LD. Hyponatremia in the prognosis of acute ischemic stroke. Journal of Stroke and Cerebrovascular Disease. 2014; 23 (5): 850-54.

[15] Soiza RL, Cumming K, Clark AB, Bettencourt - Silva JH, Metcalf AK, Bowles KM, Potter JF, Myint PK. Hyponatremia predicts mortality after stroke. International Journal of Stroke. 2015; 10: 50-5. 